\title{
Biostatistical and mathematical models in the analysis of antimicrobial resistance due to antibiotic treatment
}

\begin{abstract}
The human microbiome is one of the most densely populated ecosystems known to man. Due to this density, the close proximity of cells in the human gut favors certain mechanisms of acquiring antimicrobial resistance (AMR), e.g., mutation and horizontal gene transfer (HGT); AMR via HGT is referred to herein as hAMR (antimicrobial resistance [developed] via horizontal gene transfer). In addition, the human microbiome is an open system meaning that, moment-to-moment, the human microbiome may be involved in encounters which can result in large populations of diverse cells becoming singularly resistant or cluster resistant to antibiotics. Devising biostatistical and mathematical models to describe the relationship of components within a system is practical and advantageous. Models represent the system being observed and allow for the analysis of the components upon that system, and to predict outcomes. From such models predictions can be made and communicated directly.
\end{abstract}

Volume 7 Issue 2 - 2018

\author{
Nicholas A Kerna ${ }^{1,2}$ \\ 'College of Medicine, University of Science,Arts and Technology, \\ USA \\ 2Department of Biostatistics and Epidemiology, Suriwongse \\ Medical Center,Thailand
}

\author{
Correspondence: Nicholas A Kerna, College of Medicine, \\ University of Science, Arts and Technology, 4288 Young Mield \\ Street, Wheat Ridge, CO 80033, USA, Email \\ nicholas.kerna@usat.edu
}

Received: March 21, 2018 | Published: April 03, 2018

Keywords: antibiotic resistance, applied mathematics, biostatistics, combinatorics, horizontal gene transfer, human microbiome, mathematical models, mutation, probability

Abbreviations: AMR, antimicrobial resistance; hAMR, antimicrobial resistance via horizontal gene transfer; HGT, horizontal gene transfer

\section{Introduction}

Antimicrobial resistance (AMR) and the lack of novel antibiotics is one of the greatest threats to the human population and to many of the medical advances that use antibiotics to augment these procedures. In the most simplistic terms, resistance to antibiotics will result in more untreatable illnesses and diseases, accelerating mortality rates. This will increase the cost of patient care and tax the already overburdened healthcare system; and will impact families, employers and society as a whole.

How AMR affects treatment and outcome in certain bacterial infections can be described in a narrative form, such as: elevated AMR increases disease and death. But that story reveals little about other cofactors; and how adjusting one factor may affect another factor, several or all factors. It is more useful to develop a mathematical model depicting these cofactors and their relationships; in this case to more readily recognize and more easily assess the effects of AMR on sickness and mortality.

\section{Discussion}

A biostatistical model can determine if variables are related and, according to the data, compute probability distribution; and can reveal if the variables are related in a statistically significant way-dependent and independent variables. This results in a mathematical equation, but falls short of being a mathematical model. A mathematical model will consider and incorporate other formulas and determinants into the system, such as Combinatorics and Probability. The mathematical model helps explain a system and the relationships of the factors, and in making predictions. The model will allow for probabilities and proportions to be determined. Integrating biostatistical data into a mathematical model will result in a more realistic and dynamic paradigm. Developing a model(s) to depict the effect of AMR on mortality is presumed a constructive endeavor; as it is estimated that ten million people die per year due complications related to AMR.

\section{Conclusion}

The implementation of biomedical research regarding an Antibiotic-AMR-Mortality-Rate Model is needed. This includes how bacteria evolve and diversify; DNA and genetic coding are elemental to this research. Biostatistical and mathematical modeling are tools that provide meaningful data and interpretive formulations. The integration of biostatistical and mathematical models can explicate more distinct findings, conclusions and projections regarding the escalating antimicrobial resistance crisis.

\section{Acknowledgements}

None.

\section{Conflicts of interest statement}

The author declares that this research was conducted in the absence of any commercial or financial relationships that could be construed as a potential conflict of interest.

\section{Bibliography}

1. Antibiotics. Grace Communication Foundation. http://www. sustainabletable.org/257/antibiotics. Accessed January 15, 2018.

2. Campinas. Mathematical Modeling of microbial growth in milk. Food Science and Technology. http://www.scielo.br/scielo.php?script=sci arttext\&pid=S0101-20612011000400010. Published Oct./Dec. 2011. Accessed January 25, 2018. 
3. Heffner. CL. Types of Distribution. AllPsch. http://allpsych.com/ researchmethods/distributions/. Accessed January 15, 2018.

4. Rapid Learning. Density Curves. http://www.rapidlearningcenter.com/ mathematics/introductory-statistics/07-Density-Curves.html. Accessed January 15, 2018.

5. Stephanie. Statistics How to. http://www.statisticshowto.com/skewness/. Published September 23, 2013. Updated March 17, 2018. Accessed March $18,2018$.
6. West Texas A\&M University. Graphs and Functions. http://www.wtamu. edu/academic/anns/mps/math/mathlab/col_algebra/col_alg_tut31 graphfun1.htm. Revised April 7, 2010. Accessed January 15, 2018. 